James Madison University JMU Scholarly Commons

Masters Theses

The Graduate School

Spring 2014

\title{
The influence of a CYP1A2 polymorphism on the hemostatic response to caffeine
}

Joseph James Aiello

James Madison University

Follow this and additional works at: https://commons.lib.jmu.edu/master201019

Part of the Kinesiology Commons

\section{Recommended Citation}

Aiello, Joseph James, "The influence of a CYP1A2 polymorphism on the hemostatic response to caffeine" (2014). Masters Theses. 127. https://commons.lib.jmu.edu/master201019/127

This Thesis is brought to you for free and open access by the The Graduate School at JMU Scholarly Commons. It has been accepted for inclusion in Masters Theses by an authorized administrator of JMU Scholarly Commons. For more information, please contact dc_admin@jmu.edu. 
The Influence of a CYP1A2 Polymorphism on the Hemostatic Response to Caffeine Joseph James Aiello

A thesis submitted to the Graduate Faculty of JAMES MADISON UNIVERSITY

In

Partial Fulfillment of the Requirements

for the degree of

Master of Science

Exercise Physiology

May 2014 


\section{Dedication}

I would like to dedicate my thesis to my loving Grandmother, Ann Robbie. Her unconditional support throughout my time as both an undergraduate and graduate student has been nothing but a blessing. Her grandchildren are truly cherished and never without her thoughts and prayers, something I will never take for granted.

I would also like to dedicate this thesis to my father, Joe. Without his values, humor, and most importantly his work ethic, I would never have made it this far in my career. His support in my pursuit of my own passion has allowed me to develop into the man that I am today and I am eternally grateful for that. To my siblings, Laura and Andrew, for their loving support and commitment to their oldest brother.

Finally, and certainly not least, I would like to dedicate this piece of work to my mother, Ann Marie. Her unremitting concern for my well-being and academic status has been one of the few reasons I have been able to maintain the commitment to my studies. I am sincerely thankful to have someone like you in my life. No matter how tough it gets, my family has been the most resilient group of people I have come to know and it is because of this quality, that I have learned to overcome every and any obstacle that appears in my path to success. Thank you all for your love and support. 


\section{Acknowledgements}

I wish to thank my committee members for all of the expertise and precious time they have shared in the writing process of this thesis. A special thanks to my committee chairman, Dr. Womack. Throughout this project it has become quite clear how lucky I am to have been able to work underneath you. Your advice and concern throughout the project has given me a newfound respect for the field of Exercise Physiology. I would like to thank Dr. Saunders and Dr. Hargens for agreeing to be a part of my thesis committee and all of the help they have given along the way.

Additionally, I would like to acknowledge all of the undergraduate Kinesiology majors that assisted throughout the entire process of our trials. Your dedication to being on-time and mentally prepared during the early and extensive hours of the day allowed this project to meet its full potential Thank you Liz Zwicker, Dan Shill, Michael Kayajanian, and Mitchell Bowers.

Finally, I would like to acknowledge the Human Performance Lab and its members at James Madison University for allowing our project to be added to their wide range of highly regarded body of work in exercise physiology. 


\section{Table of Contents}

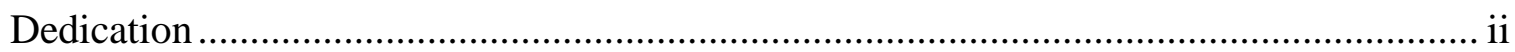

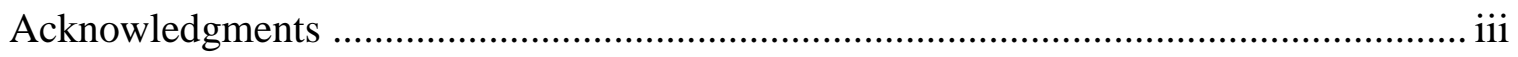

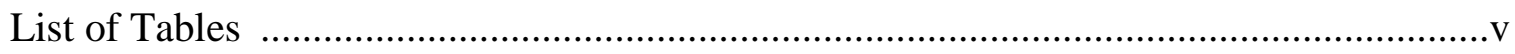

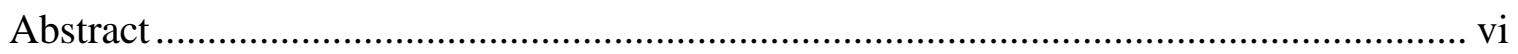

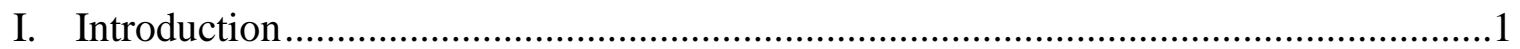

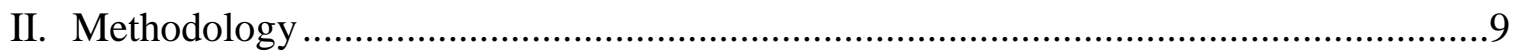

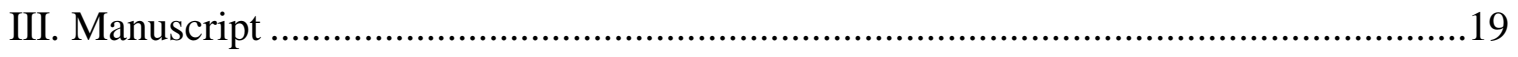




\section{List of Tables}

Table 1. Descriptive Characteristics

Table 2: Markers of Coagulation Potential

2A. Plasma Factor VIII before and after exercise in both treatments for both genetic groups.

2B. Plasma Fibrinogen before and after exercise in both treatments for both genetic groups.

Table 3: Markers of Fibrinolytic Potential

3A. Plasma Tissue Plasminogen Activator (tPA) activity before and after exercise in both treatments for both genetic groups.

3B. Plasma tPA antigen before and after exercise in both treatments for both genetic groups.

3C. Plasma Plasminogen Activator Inhibitor- 1 (PAI-1) before and after exercise in both treatments for both genetic groups. 


\begin{abstract}
Research has continued to provide conflicting results of caffeine's effect on acute coronary events; although it is now apparent that a single nucleotide polymorphism at intron 1 of the cytochrome P450 (CYP1A2) gene may explain these equivocal findings. Despite the fact that markers of coagulation potential and fibrinolytic potential are associated with risk for myocardial infarction, the extent to which caffeine affects these markers is unknown. The purposes of this study were to determine the acute effects of caffeine on coagulation and fibrinolytic potential at rest and following maximal exercise and to determine if hemostatic responses engendered by caffeine are influenced by the aforementioned CYP1A2 polymorphism. 20 healthy, recreationally active, males (age, 21.4 \pm 1.2 SD; weight, $81.9 \pm 10.4 \mathrm{SD} ; 180.6 \pm 7.1 \mathrm{SD} ; \mathrm{VO}_{2 \text { peak }} 46.4 \pm 6.17 \mathrm{SD}$ $\mathrm{mL} \cdot \mathrm{kg} \cdot \mathrm{min}^{-1}$ ) participated in this study. Subjects performed two graded maximal exercise tests (GXT's) after consuming either $6 \mathrm{mg} / \mathrm{kg}$ of caffeine or placebo 1 hour before trials. DNA was obtained from whole blood samples and genotyping was done using restriction fragment length polymerase chain reaction. Subjects were categorized as possessing the $\mathrm{C}$ allele ( $\mathrm{C}$ allele carriers) or being homozygous for the $\mathrm{A}$ allele (AA homozygotes). The effects of treatment (caffeine, placebo), time (pre-exercise, post-exercise), and treatment x genotype were assessed using Repeated Measures Analysis of Variance (RMANOVA). Caffeine was shown to increase plasma Factor VIII levels when compared to placebo, but this was no longer significant when covarying for the increased exercise time engendered by caffeine. Fibrinogen values significantly increased post-exercise $(\mathrm{p}<0.05)$ for $\mathrm{C}$ allele carriers but not for AA homozygotes. Finally, caffeine caused a significantly higher resting plasma tPA activity and a larger tPA response to exercise. Our results
\end{abstract}


suggest that caffeine may increase Factor VIII response to graded exercise (GXT) secondary to an effect on time to fatigue (TTF). Caffeine also appears to increase fibrinolytic potential at rest and after exercise. Additionally, the aforementioned CYP1A2 polymorphism appears to impact the fibrinogen response to exercise. 


\section{Chapter One}

Introduction

There has been conflicting research related to caffeine's effect on cardiovascular disease, serum lipids, and acute coronary events [6,12,16,29,33,34,,44,45,48, 53]. Current literature shows both $\mathrm{J}$ and $\mathrm{U}$-shaped relationships between cardiovascular disease risk and coffee consumption, suggesting a type of protective effect for moderate, but not light or heavy drinkers $[29,36,48]$. Furthermore, numerous studies evaluating the consumption of caffeinated versus decaffeinated coffee show preventative cardiovascular benefits $[13,34,41]$ while others show an increased relative risk for acute coronary events like myocardial infarction and stroke $[29,48,53]$. It has also been suggested that coffee consumption may acutely trigger a myocardial infarct in sedentary individuals that drink less than 2 cups of coffee per day or those whom do not consume coffee daily [6]. Happonen et al observed a dose-dependent relationship for risk of acute myocardial infarction [6], while Kim et al found that daily consumption of 4 or more cups of coffee per day may elicit a protective effect for stroke [53].

The association of cardiovascular disease with coffee consumption has been investigated for several decades, yet findings have obviously been equivocal. As an example, a prior meta-analysis included findings from 22 cohort and case-control studies from 1968 to 1992 . Half of the cohort studies observed a relative risk ratio of 1.3-1.5 for acute myocardial infarction with the consumption of at least 5 cups of coffee per day; while the other half showed no increased risk of such events [25]. Similarly, an analysis of another 23 case-control and cohort studies found that the cohort studies reported no 
additional risk of CHD whereas the case-control studies resulted in a $60 \%$ increase in risk in those who consume 5 cups of coffee per day or more [34]. However, after further investigation, it has been noted that a majority of these analyses did not control for other variables that may influence the relative risk of cardiovascular disease such as smoking status, habitual alcohol consumption, and dietary factors [12,25,33,34]. Because of this, Sofi and colleagues controlled for multiple variables including age, gender, smoking status, history of MI, and, physical activity [54]. The meta-analysis showed a risk ratio of 1.83 in drinkers that consumed more than 4 cups of coffee per day for Coronary Heart Disease in the case-control studies analyzed. In contrast, the long-term follow-up cohort studies showed no additional risk of CHD with any level of coffee consumption [54].

A more recent study evaluated the results of the Nurses' Health Study and Health Professionals Follow-Up Study which included bi-annual reports of coffee consumption and health outcomes of more than 120,000 male and female participants over a period of 34 years. After correcting for smoking status, a significant inverse association was found between coffee consumption and all-cause deaths, suggesting a positive health benefit from coffee consumption [41]. In addition, among women, 5 to 7 cups of coffee per day resulted in a $7 \%$ reduced risk of death compared to non-drinkers. Furthermore, a reduction in all-cause death was observed across levels of coffee consumption, with a reported $18 \%$ reduction in those drinking 2-3 cups per day, 26\% drinking 4-5 cups per day, and $17 \%$ drinking $6+$ cups per day. These findings support the notion that coffee consumption in women may exhibit a protective effect in moderate drinkers [48]. Additionally, the National Institute of Health - AARP Diet and Healthy Study evaluated 229,119 men and 173,141 women between the ages of 50 and 71. After correcting for 
smoking, an inverse association was observed between every level of coffee consumption and specific-cause mortality rate related to heart disease, stroke, respiratory disease, and diabetes. [23].

In contrast, a recent study by Liu and colleagues analyzed deaths during a 17 year period in 43,727 participants from the Aerobics Center Longitudinal Study. A multivariate analysis showed a positive association between coffee intake and all-cause mortality in men [40]. When adjusted for age, a significant association between high coffee consumption and all-cause mortality was observed for both men and women younger than 55. In addition, a positive relationship between coffee and blood lipids was observed by Cai et al. The group analyzed 12 randomized controlled trials performed that included coffee drinking as the active treatment intervention. All 12 studies monitored total cholesterol, LDL, HDL, and TGs over a period of at least 45 days [12]. LDL and triglyceride levels increased and this effect was more pronounced in those who were previously diagnosed for hyperlipidemia $[12,32]$. Studies investigating the effect of coffee on serum lipids have yielded similar results in addition to an observed increase in inflammatory markers $[18,55]$.

While the literature in regards to coffee consumption and cardiovascular risk remains ambiguous at best, the effect of caffeine on hemodynamic response is unequivocal. Caffeine has been known to increase heart rate, blood pressure, myocardial contractility, and cardiac output $[7,12,13,2933,34,44,45,49]$. Prior data shows that contractility significantly increases 90 minutes after ingestion, and does not return to baseline until 4.5 hours after the initial dosage [7]. Caffeine's effect on heart rate is a direct response to increased catecholamine release that results in an effect similar to that 
of other stimulants $[12,19,29,33,34,44,45]$. Caffeine also increases blood pressure by inhibition of adenosine receptors as well as increased neuroendocrine stimulation [12,29,33,34,43-45]. A study on a group of healthy, non-coffee drinkers showed significant increases in blood pressure and total peripheral resistance following ingestion of 200 milligrams of caffeine [13]. Additionally, Papaioannou observed acute increases in arterial stiffness after the ingestion of caffeine [49]. These effects can be seen within 30 minutes of consumption and continue for 4 or more hours [43]. While increases in systolic pressure range between 3 and $15 \mathrm{mmHg}$, diastolic pressures can also rise 4-13 mmHg [43]. Habitual coffee consumption has been theorized to influence the effect of caffeine on the neuroendocrine and cardiovascular system. However, it has been found that tolerance minimally attenuates the corresponding responses in blood pressure, heart rate, and total peripheral resistance following caffeine consumption [22,52,57]. These acute physiological effects could be especially important in those at risk for cardiovascular disease.

Although hemodynamic responses to caffeine may lead to acute myocardial infarction, it is understood that a majority of myocardial infarctions result from an occlusive thrombus $[24,37,53]$. Unstable plaques rupture, resulting in thrombus formation that can completely occlude coronary arteries. Thus, plasma markers of coagulation potential and clot lysis (fibrinolysis) are clinically important [50,59]. Markers of coagulation potential include factors that make up the coagulation cascade and fibrinogen, the inactive precursor to fibrin. In humans, fibrinolysis is regulated by conversion of plasminogen in to plasmin which degrades fibrin to smaller fibrin dimer proteins. This conversion is primarily regulated by tissue plasminogen activator (tPA). 
The main circulating inhibitor of tPA is plasminogen activator inhibitor (PAI-1), which binds to tPA and forms an inactive complex. The hemostatic response to exercise is of particular clinical significance as $80 \%$ of exertion-related ischemic events occur due to an occlusive thrombus [24]. Exercise acutely increases coagulation potential [20,21], which is offset by a corresponding increase in fibrinolytic activity [59].

Coffee and caffeine consumption was originally linked to myocardial infarction based on its potential to induce arrhythmias, although this theory has long been discredited [19,25]. However, it is possible that acute [30] and chronic [18] caffeine intake has a role in increasing coagulation potential. In a recent study, participants were exposed to a series of cognitive tasks, including a speech challenge and timed arithmetic tasks, specifically designed to elicit stress. After the ingestion of a placebo drink or 3.3 $\mathrm{mg} / \mathrm{kg}$ of caffeine, higher levels of fibrinogen were observed following caffeine ingestion at rest, 15 minutes after exposure to the stressor, and during the recovery period [30]. In contrast, Bak et al observed that nine weeks of caffeine supplementation did not alter markers of coagulation. In this study, 107 young, healthy adults were given $75 \mathrm{mg}$ of caffeine or placebo per day for a period of 9 weeks. There were no observed effects of caffeine consumption on resting levels of factor VII, factor VIII, and fibrinogen [4]. Thus, whether or not caffeine affects hemostasis is not fully known and the interaction between caffeine intake and exercise on the hemostatic profile has not been evaluated.

The equivocal findings on the relationship between coffee intake and risk of MI, and the conflicting findings regarding caffeine's effect on hemostasis could be explained by genetic differences. Cytochrome P450 (CYP1A2) is a hepatic enzyme that is responsible for the metabolism of caffeine. Cornelius et al observed that a single- 
nucleotide $(\mathrm{A} / \mathrm{C})$ polymorphism at intron 1 of the CYP1A2 gene influenced the degree to which coffee consumption was associated with risk of myocardial infarction [17]. Coffee consumption increased $\mathrm{MI}$ risk in study participants that possessed a $\mathrm{C}$ allele, but was not linked to MI risk in individuals homozygous for the A allele. Prior research has shown that $\mathrm{C}$ allele carriers have slower caffeine metabolism than AA homozygotes [16], suggesting that the increased MI risk in C-allele carriers was due to higher plasma caffeine levels following caffeine dosage. This polymorphism has also been shown to influence the association between caffeine and bone mineral density [27] and the ergogenic response to caffeine in cyclists [60]. The former study evaluated whether the CYP1A2 polymorphism impacted the long-term effect of drinking high amounts of coffee and corresponding bone mass in an elderly population. Bone mineral density was evaluated for coffee drinkers who consumed greater than 4 cups of coffee per day and was lower in AA homozygotes genotype than in $\mathrm{C}$ allele carriers. It was hypothesized that the lower BMD may be due to caffeine's potential to increase calcium excretion and decrease absorption that can lead to lower bone mineral density. Furthermore, the lower BMD in AA homozygote may have been due to the impact of caffeine metabolites on the aforementioned effects of caffeine on BMD [27]. Womack et al observed that caffeine significantly improved cycling time trial performance in AA homozygotes to a greater degree than in C allele carriers [60]. While the mechanisms for these effects are still unknown, the implications of such findings suggest that this genetic polymorphism exerts a very powerful influence on caffeine response.

If caffeine increases risk of MI due to alterations in hemostasis, it could be hypothesized that the aforementioned CYP1A2 polymorphism would influence the effect 
of caffeine on the coagulation and/or fibrinolytic profile. The purposes of the present study are to: 1) determine the acute effects of caffeine on coagulation and fibrinolytic potential at rest and following maximal exercise and 2) determine if hemostatic responses engendered by caffeine are influenced by the aforementioned CYP1A2 polymorphism. It is hypothesized that caffeine will increase coagulation potential both at rest and after exercise and this increase will be more pronounced in C-allele carriers.

\section{Assumptions}

- $\quad$ All subjects will have a greater Resting Heart Rate from Caffeine compared to Placebo

Limitations

- $\quad$ No Females used in study

- $\quad$ All healthy subjects without previous clinical complications (CVD, Chronic Illness)

- $\quad$ All "young" healthy males

Delimitations

- $\quad$ All Tests done before 9:00 AM to control for changes in circadian hemodynamic response 
- $\quad$ All subjects were non-smokers

- $\quad$ All subjects were fasted of food at least 10 hours before each trial

- $\quad$ All subjects had no illnesses or infections at the time of testing

Definition of Terms

Markers of Coagulation:
a. Factor VIII
b. Fibrinogen

Markers of Fibrinolysis:
a. Tissue-Plasminogen Activator (tPA)
b. Tissue-Plasminogen Activator Antigen (tPA Antigen)
c. Plasminogen Activator Inhibitor (PAI-1) 


\section{Chapter Two}

Methods

\section{Subjects}

A total of 20 healthy, recreationally active, males (age, $21.4 \pm 1.2$; weight, $81.9 \pm$ $10.4 ; 180.6 \pm 7.1 ; \mathrm{VO}_{2 \text { peak }} 46.4 \pm 6.17 \mathrm{~mL} \cdot \mathrm{kg} \cdot \mathrm{min}^{-1}$ ) participated in this study. All subjects were non-smokers, recruited from a university setting without any reported chronic illness or condition. Written informed consent was obtained from all participants prior to participation. The study and consent form were approved by the James Madison University Institutional Review Board.

\section{Experimental Protocol}

The study used a randomized, double-blind, crossover design. All subjects performed a 10 hour fast on the night before each trial and refrained from the consumption of caffeine and alcohol for 24 hours prior to each trial. Exclusion criteria included any illness 7 days prior to the trial as well as the use of medication within 24 hours of any trial. Subjects performed two graded maximal exercise tests (GXT's). All trials were performed before 10:00 hours in order to minimize the possibility of diurnal variation in fibrinolysis [2]. Subjects ingested $6 \mathrm{mg} / \mathrm{kg}$ of pharmaceutical grade caffeine (Nutrabio, New Jersey) or placebo 1 hour before any tests. Caffeine was dissolved in 10 oz of a non-caloric, flavored drink while the placebo solution consisted of the drink without addition of caffeine. After 45 minutes of ingestion, subjects were placed in a semi-recumbent position and rested for 15 minutes to minimize the effect of postural 
changes on fibrinolysis [58]. At exactly 60 minutes post-ingestion, a pre-exercise blood sample (10-ml) was taken. Blood samples were collected in tubes containing an acidified citrate solution (Tcoag Ireland Limited; Bray Co. Wicklow Ireland) for tPA and PAI-1 activity measurement and in citrate tubes for the analysis of FVIII, tPA Antigen, and Fibrinogen.

Immediately after the blood draw, subjects began a graded exercise test. All tests were performed on a VIAsprint 150P (Sensormedics, California) electronically braked cycle ergometer. The test began at 25 watts and increased 25 watts every minute until volitional fatigue or the subject failed to maintain 60 RPMs for more than 5 consecutive seconds. Oxygen uptake and heart rate was measured continuously using a ParvoMedics TrueOne Metabolic Cart (Sandy, UT) and at every stage using a Polar Heart Rate Monitor (Lake Success, NY), respectively. $\mathrm{VO}_{2}$ peak was defined as the highest 30second average obtained during the test. Once the trial was finished, subjects were immediately placed in a semi-recumbent position and a post-exercise blood sample (10ml) was taken. All post-exercise samples were obtained within 2 minutes to eliminate potential changes in post-exercise fibrinolytic variables [15].

\section{Genotyping}

Investigators were blinded to genotype until all subjects completed the study. Furthermore, all genotyping was performed by an investigator not involved with the exercise testing. DNA was obtained from whole blood samples via an illustra blood genomic prep mini spin kit (GE Healthcare Life Sciences.; Buckinghamshire, UK). Each blood sample was obtained prior to one of the cycling trials. Genotyping was performed 
using restriction fragment length polymerase chain reaction (RFLP-PCR), as previously described [16]. Subjects were categorized as possessing the $\mathrm{C}$ allele ( $\mathrm{C}$ allele carriers) or being homozygous for the A allele (AA homozygotes).

\section{Assays}

Blood samples were centrifuged at $4000 \mathrm{~g} / \mathrm{min}$ at $4{ }^{\circ} \mathrm{C}$ for $20 \mathrm{~min}$ to obtain platelet-poor plasma. Plasma samples were analyzed using an enzyme-linked immunosorbancy assay (ELISA) for Factor VIII (Affinity Biologicals; Ontario, CA), Tissue Plasminogen Activator (tPA) (Eagle; Nashua,NH), Tissue Plasminogen Antigen (Eagle; Nashua, NH), Plasminogen Activator Inhibitor -1 (PA-1)(Molecular Innovations; Novi, MI). Researchers at Ball State University performed analysis of fibrinogen via the use of a coagulometer.

\section{Statistical Analysis}

All statistical analysis were conducted using SPSS software (Chicago, IL). A three-factor Repeated Measures analysis of variance (RMANOVA) test was ran with treatment (caffeine, placebo) and time (pre-exercise, post-exercise) as within-subjects factors and genotype (AA homozygotes, $\mathrm{C}$ allele carriers) as a between-subjects factor. Potential differences in time to fatigue and $\mathrm{VO}_{2 \text { peak }}$ were analyzed using a RMANOVA with treatment as the within-subjects factor and genotype as the between-subjects factor. For all RMANOVA tests, post-hoc interactions were performed using paired and unpaired t-tests. A P-value of $<0.05$ was considered statistically significant. 


\section{Chapter Three}

\section{Manuscript}

There has been conflicting research related to caffeine's effect on cardiovascular disease, serum lipids, and acute coronary events [6,12,16,29,33,34,44,45,48,53]. Current literature shows both $\mathrm{J}$ and $\mathrm{U}$-shaped relationships between cardiovascular disease risk and coffee consumption, suggesting a type of protective effect for moderate but not light or heavy drinkers $[29,36,48]$. Furthermore, numerous studies evaluating the consumption of caffeinated versus decaffeinated coffee show preventative cardiovascular benefits regardless of type $[13,34,41]$ while others show an increased relative risk for acute coronary events like myocardial infarction and stroke [29,48,53]. It has also been suggested that coffee consumption may acutely trigger a myocardial infarct in sedentary individuals that drink less than 2 cups of coffee per day or those not consuming coffee daily [6].

While the literature in regards to coffee consumption and cardiovascular risk remains ambiguous at best, the effect of caffeine on hemodynamic response is unequivocal. Caffeine increases heart rate, blood pressure, myocardial contractility, and cardiac output $[7,12,13,29,33,34,44,45,49]$. Prior data shows that contractility increases 90 minutes after ingestion, and does not return to baseline until 4.5 hours after the initial dosage [7]. Caffeine's effect on heart rate is a direct response to increased catecholamine release that results in an effect similar to that of other stimulants $[12,19,29,33,34,44,45]$. Caffeine also increases blood pressure by inhibition of adenosine receptors, as well as 
increased neuroendocrine stimulation [12,29,33,34,43-45]. These effects can be seen within 30 minutes of consumption and continue for 4 or more hours [43].

Although hemodynamic responses to caffeine may lead to acute myocardial infarction, it is understood that a majority of myocardial infarctions result from an occlusive thrombus $[24,37,53]$. Unstable plaques rupture, resulting in thrombus formation that can completely occlude coronary arteries. Thus, plasma markers of coagulation potential and clot lysis (fibrinolysis) are clinically important [50,59]. Markers of coagulation potential include factors that make up the coagulation cascade and fibrinogen, the inactive precursor to fibrin. In humans, fibrinolysis is regulated by conversion of plasminogen in to plasmin which degrades fibrin to smaller fibrin dimer proteins. The hemostatic response to exercise is of particular clinical significance as $80 \%$ of exertion-related ischemic events occur due to an occlusive thrombus [24]. Exercise acutely increases coagulation potential [20,21], which is offset by a corresponding increase in fibrinolytic activity [59].

The equivocal findings on the relationship between coffee intake and risk of MI, and the conflicting findings regarding caffeine's effect on hemostasis could be explained by genetic differences. Cytochrome P450 (CYP1A2) is a hepatic enzyme that is responsible for the metabolism of caffeine. Cornelius et al observed that a singlenucleotide $(\mathrm{A} / \mathrm{C})$ polymorphism at intron 1 of the CYP1A2 gene influenced the degree to which coffee consumption was associated with risk of myocardial infarction [17]. Coffee consumption increased MI risk in study participants that possessed a $\mathrm{C}$ allele, but was not linked to MI risk in individuals homozygous for the A allele. Prior research has shown that $\mathrm{C}$ allele carriers have slower caffeine metabolism than AA homozygotes [16], 
suggesting that the increased MI risk in C-allele carriers was due to higher plasma caffeine levels following caffeine dosage. This polymorphism has also been shown to influence the association between caffeine and bone mineral density [27] and the ergogenic response to caffeine in cyclists [60]. The former study evaluated whether the CYP1A2 polymorphism impacted the long-term effect of drinking high amounts of coffee and corresponding bone mass in an elderly population. Womack et al observed that caffeine significantly improved cycling time trial performance in AA homozygotes to a greater degree than in $\mathrm{C}$ allele carriers [60]. While the mechanisms for these effects are still unknown, the implications of such findings suggest that this genetic polymorphism exerts a very powerful influence on caffeine response.

If caffeine increases risk of MI due to alterations in hemostasis, it could be hypothesized that the aforementioned CYP1A2 polymorphism would influence the effect of caffeine on the coagulation and/or fibrinolytic profile. The purposes of the present study were to: 1) determine the acute effects of caffeine on coagulation and fibrinolytic potential at rest and following maximal exercise and 2) determine if hemostatic responses engendered by caffeine are influenced by the aforementioned CYP1A2 polymorphism. It is hypothesized that caffeine will increase coagulation potential both at rest and after exercise and this increase will be more pronounced in C-allele carriers. 
Methods

\section{Subjects}

A total of 20 healthy, recreationally active, males (age, $21.4 \pm 1.2$; weight, $81.9 \pm$ $10.4 ; 180.6 \pm 7.1 ; \mathrm{VO}_{2 \text { peak }} 46.4 \pm 6.17 \mathrm{~mL} \cdot \mathrm{kg} \cdot \mathrm{min}^{-1}$ ) participated in this study. All subjects were non-smokers and were recruited from a university setting without any reported chronic illness or condition. Written informed consent was obtained from all participants prior to participation. The study and consent form were approved by the James Madison University Institutional Review Board.

\section{Experimental Protocol}

The study used a randomized, double-blind, crossover design. All subjects performed a 10 hour fast on the night before each trial and refrained from the consumption of caffeine and alcohol for 24 hours prior to each trial. Exclusion criteria included any illness 7 days prior to the trial as well as the use of medication within 24 hours of any trial. Subjects performed two graded maximal exercise tests (GXT's). All trials were performed before 10:00AM in order to minimize the possibility of diurnal variation in fibrinolysis [2]. Subjects ingested $6 \mathrm{mg} / \mathrm{kg}$ of pharmaceutical grade caffeine (Nutrabio, New Jersey) or placebo 1 hour before any tests. Caffeine was dissolved in 10 oz of a non-caloric, flavored drink while the placebo solution consisted of the drink without addition of caffeine. After 45 minutes of ingestion, subjects were placed in a semi-recumbent position and rested for 15 minutes to minimize the effect of postural changes on fibrinolysis [58]. At exactly 60 minutes post-ingestion, a pre-exercise blood 
sample (10-ml) was taken. Blood samples were collected in tubes containing an acidified citrate solution (Tcoag Ireland Limited; Bray Co. Wicklow Ireland) for tPA and PAI-1 activity measurement and in regular citrate tubes for the analysis of FVIII, tPA Antigen, and Fibrinogen.

Immediately after the blood draw, subjects began a graded exercise test. All tests were performed on a VIAsprint 150P (Sensormedics, California) electronically braked cycle ergometer. The test began at 25 watts and increased 25 watts every minute until volitional fatigue or the subject failed to maintain 60 RPMs for more than 5 consecutive seconds. Oxygen uptake and heart rate was measured continuously using a ParvoMedics TrueOne Metabolic Cart (Sandy, UT) and at every stage using a Polar Heart Rate Monitor (Lake Success, NY), respectively. $\mathrm{VO}_{2 \text { peak }}$ was defined as the highest 30 -second average obtained during the test. Once the trial was finished, subjects were immediately placed in a semi-recumbent position and a post-exercise blood sample (10-ml) was taken. All post-exercise samples were obtained within 2 minutes to eliminate potential changes in post-exercise fibrinolytic variables [15].

\section{Genotyping}

Investigators were blinded to genotype until all subjects completed the study. Furthermore, all genotyping was performed by an investigator not involved with the exercise testing. DNA was obtained from whole blood samples via an Illustra blood genomic prep mini spin kit (GE Healthcare Life Sciences.; Buckinghamshire, UK). Each blood sample was obtained prior to one of the cycling trials. Genotyping was performed using restriction fragment length polymerase chain reaction (RFLP-PCR), as previously 
described [16]. Subjects were categorized as possessing the $\mathrm{C}$ allele ( $\mathrm{C}$ allele carriers) or being homozygous for the A allele (AA homozygotes).

Assays

Blood samples were centrifuged at $4000 \mathrm{~g} / \mathrm{min}$ at $4{ }^{\circ} \mathrm{C}$ for $20 \mathrm{~min}$ to obtain platelet-poor plasma. Plasma samples were analyzed using an enzyme-linked immunosorbancy assay (ELISA) for Factor VIII (Affinity Biologicals; Ontario, CA), Tissue Plasminogen Activator (tPA) (Eagle; Nashua,NH), Tissue Plasminogen Antigen (Eagle; Nashua, NH), Plasminogen Activator Inhibitor -1 (PA-1)(Molecular Innovations; Novi, MI). Researchers at Ball State University performed analysis of fibrinogen via the use of a coagulometer.

\section{Statistical Analysis}

All statistical analysis were conducted using SPSS software (Chicago, IL). A three-factor Repeated Measures analysis of variance (RMANOVA) test was performed with treatment (caffeine, placebo) and time (pre-exercise, post-exercise) as within-subjects factors and genotype (AA homozygotes, $\mathrm{C}$ allele carriers) as a between-subjects factor. Potential differences in time to fatigue and $\mathrm{VO}_{2 \text { peak }}$ were analyzed using a RMANOVA with treatment as the within-subjects factor and genotype as the between-subjects factor. For all RMANOVA tests, post-hoc interactions were performed using paired and unpaired ttests. A P-value < 0.05 was considered statistically significant. All variables are expressed as mean, plus or minus standard deviation. 
Results

Of the 20 subjects, $10(50 \%)$ were homozygous for the A allele and $10(50 \%)$ were $\mathrm{C}$ allele carriers. This distribution is similar to previously reported studies regarding the presence of the polymorphism in a sample population $[16,17,60]$. Descriptive statistics between the two genetic groups are displayed in Table 1. No significant differences were observed between AA homozygotes and $\mathrm{C}$ allele carriers for age, weight, height, or $\mathrm{VO}_{2 \text { peak}}$. There was a trend $(\mathrm{p}=$ $0.09)$ for increased time to fatigue in the caffeinated $(711 \pm 139 \mathrm{sec})$ versus the placebo $(687 \pm$ $112 \mathrm{sec})$ conditions.

Table 2 shows pre and post-exercise values for markers of coagulation potential. There were significant main effects for both treatment (Caffeine $>$ Placebo, $\mathrm{p}<0.05)$ and time (PostExercise $>$ Pre-Exercise, $\mathrm{p}<0.05$ ) for plasma levels of Factor VIII (Table 2A). Factor VIII increased by $189 \%$ during exercise in the caffeinated condition as compared to $131 \%$ during exercise in the placebo condition, although there was not a significant treatment $\mathrm{x}$ time interaction $(p=0.10)$. Because of the trend for increased TTF in the caffeine trial, all ANOVA analyses were repeated while covarying for the difference in TTF during the caffeine trial and TTF in the placebo trial (delta TTF). As a result of this post-hoc analysis, there was no longer a significant effect of treatment on plasma Factor VIII. None of the other findings were altered. Plasma fibrinogen values are shown inTable $2 \mathrm{~B}$. A significant treatment $\mathrm{x}$ time $\mathrm{x}$ genotype interaction was observed due to a $23 \%$ increase $(\mathrm{p}<0.05)$ during exercise in the caffeinated condition for the $\mathrm{C}$ allele carriers. No significant increase in fibrinogen was observed during exercise in the caffeinated condition for the AA homozygotes and no increases were observed for either group during exercise in the placebo condition. 
Values for fibrinolytic variables are displayed in Table 3. There were significant main effects for both treatment (Caffeine $>$ Placebo, $\mathrm{p}<0.05)$ and time (Post-Exercise $>$ Pre-Exercise, $\mathrm{p}<0.05)$ for plasma tPA activity $($ Table $3 \mathrm{~A})$. There was also a significant treatment $\mathrm{x}$ time interaction $(\mathrm{p}<0.05)$ due to a larger increase in plasma tPA during exercise in the caffeinated condition. Additionally, a significant time $\mathrm{x}$ genotype interaction $(\mathrm{p}<0.05)$ was observed as $\mathrm{C}$ allele carriers exhibited higher tPA activity post-exercise independent of treatment. A significant main effect of time (Post-Exercise $>$ Pre-Exercise, $\mathrm{p}<0.05)$ was observed for tPA antigen (Table 3B) although no significant effects of treatment, genotype, or any interaction effects were observed. No main effects or interaction effects were observed for plasma PAI-1 (Table 3C). 
Table 1. Descriptive Characteristics

\begin{tabular}{|llll|}
\hline & AA $(\mathrm{n}=10)$ & $\mathrm{C}(\mathrm{n}=10)$ & Total $(\mathrm{n}=20)$ \\
Age & $21.1 \pm 1.2$ & $21.7 \pm 1.3$ & $21.4 \pm 1.27$ \\
Weight $(\mathrm{kg})$ & $81.3 \pm 9.4$ & $82.6 \pm 11.7$ & $81.9 \pm 10.4$ \\
Height $(\mathrm{cm})$ & $182.9 \pm 5.7$ & $179.1 \pm 8.2$ & $180.6 \pm 7.1$ \\
$\mathrm{VO}_{2 \text { peak }}\left(\mathrm{mL} \cdot \mathrm{kg}^{-1} \cdot \mathrm{min}^{-1}\right)$ & $47.0 \pm 7.0$ & $45.9 \pm 5.5$ & $46.5 \pm 6.2$ \\
\hline
\end{tabular}


Table 2: Markers of Coagulation Potential

A. Plasma Factor VIII before and after exercise in both treatments for both genetic groups.

\begin{tabular}{|c|c|c|}
\hline Treatment/Group & Pre-Exercise & Post-Exercise \\
\hline \multicolumn{3}{|l|}{ Caffeine* } \\
\hline AA & $1.034 \pm .461$ & $2.642 \pm 1.50$ \\
\hline C & $0.822 \pm .442$ & $2.691 \pm .933$ \\
\hline \multicolumn{3}{|l|}{ Placebo } \\
\hline AA & $1.06 \pm .434$ & $2.057 \pm 1.14$ \\
\hline C & $0.83 \pm .335$ & $2.228 \pm 1.03$ \\
\hline
\end{tabular}

*- Main effect for Treatment (Caffeine $>$ Placebo, $\mathrm{p}<0.05$ ); $\uparrow$ - Main effect for Time (PostExercise $>$ Pre-Exercise, $\mathrm{p}<0.05$ )

B. Plasma Fibrinogen before and after exercise in both treatments for both genetic groups.

\begin{tabular}{|lcc|}
\hline Treatment/Group & Pre-Exercise & Post-Exercise \\
Caffeine & \\
AA & $343.07+163.1$ & $345.87+130.7$ \\
C & $305.56+73.4$ & $376.2+126.3^{* *}$ \\
Placebo & \\
AA & $330.53+172.7$ & $400.0+158.2$ \\
C & $329.54+120.9$ & $329.56+105.3$ \\
\hline
\end{tabular}

**-Significantly greater than pre-exercise $(\mathrm{p}<0.05)$ 
Table 3: Markers of Fibrinolytic Potential

A. Plasma Tissue Plasminogen Activator (tPA) activity before and after exercise in both treatments for both genetic groups.

\begin{tabular}{|lcc|}
\hline Treatment/Group & Pre-Exercise & ${\text { Post-Exercise }{ }^{\mathrm{a}}}^{\mathrm{a}}$ \\
Caffeine* $^{*}$ & & $8.234+4.3$ \\
$\mathrm{AA}$ & $0.342+0.2$ & $11.285+3.2$ \\
$\mathrm{C}^{\mathrm{a}}$ & $0.428+0.3$ & \\
Placebo & & $5.391+3.0$ \\
AA & $0.263+0.2$ & $8.763+3.0$ \\
C & $0.414+0.3$ & \\
\hline
\end{tabular}

*- Main effect for Treatment (Caffeine > Placebo, $\mathrm{p}<0.05)$; $\uparrow$ - Main effect for Time (PostExercise $>$ Pre-Exercise, $\mathrm{p}<0.05)$; \#-Significant treatment $\mathrm{x}$ time interaction, with the caffeinated condition exhibiting a higher exercise response $(\mathrm{p}<0.05)$; ${ }^{\mathrm{a}}$-Significant time $\mathrm{x}$ genotype interaction, with $\mathrm{C}$ allele carriers exhibiting a higher response to exercise $(\mathrm{p}<0.05)$. 
B. Plasma tPA antigen before and after exercise in both treatments for both genetic groups.

\begin{tabular}{|lcc|}
\hline Treatment/Group & Pre-Exercise & Post-Exercise $\dagger$ \\
Caffeine & $1.124+1.3$ & $2.552+3.3$ \\
AA & $4.026+5.8$ & $6.442+8.1$ \\
C & & \\
Placebo & $1.038+1.2$ & $2.289+3.1$ \\
AA & $3.987+5.8$ & $5.812+7.4$ \\
C & & \\
\hline
\end{tabular}

$\dagger$ - Main effect for time (Post-Exercise $>$ Pre-Exercise, $\mathrm{p}<0.05$ )

C. Plasma Plasminogen Activator Inhibitor- 1 (PAI-1) before and after exercise in both treatments for both genetic groups.

\begin{tabular}{|lcc|}
\hline Treatment/Group & Pre-Exercise & Post-Exercise \\
Caffeine & & \\
AA & $9.031+11.0$ & $8.398+9.5$ \\
C & $4.774+3.5$ & $4.821+5.7$ \\
Placebo & & $7.378+10.3$ \\
AA & $7.578+10.8$ & $7.106+8.0$ \\
C & $9.223+9.1$ & \\
\hline
\end{tabular}




\section{Discussion}

In the present study, we observed an increase in plasma Factor VIII following caffeine ingestion. The process of blood clot formation, or thrombosis, is preceded by several endothelial and luminary processes which can be enhanced by the increased presence of plasma clotting factors $[1,38,46,50,59]$. Factor VIII is a part of the intrinsic pathway of the coagulation cascade, the main pathway activated during exercise-induced stimulation of coagulation, and prior studies have consistently observed increases in this marker in response to exercise [50]. There was a main effect for Factor VIII levels post-exercise $(\mathrm{p}<0.05)$ which is consistent with the current body of literature regarding post-exercise coagulation potential [50,59]. Thus, the observed increases in Factor VIII may have clinical significance given the fact that thrombosis is responsible for the majority of exertion related ischemic events [24]. It should also be noted that the effect of caffeine on Factor VIII response appeared to be more pronounced post-exercise, although there was no significant for a treatment $\mathrm{x}$ time interaction $(\mathrm{P}=0.10)$. Caffeine has been linked with ischemic events $[6,44,53]$ and the additional increase in Factor VIII from caffeine in combination with strenuous physical activity implies a potential mechanism for this relationship. These findings were not found to be mediated by genotype.

There was a trend $(p=0.09)$ for increased Time to Fatigue $(T T F)$ in the caffeinated trials. Interestingly, it appears that time to fatigue also influenced the increased Factor VIII response in the caffeinated subjects. The TTF difference between the caffeinated and placebo trials was significantly correlated $(\mathrm{R}=0.49, \mathrm{p}=0.03)$ with the magnitude of increase in Factor VIII levels when subjects ingested the caffeine supplement. Repeating the RMANOVA while covarying for TTF eliminated the observed main effect of treatment on Factor VIII. This suggests that caffeine's effect on post-exercise Factor VIII levels is a secondary outcome due to its ergogenic 
effect. Because of this particular finding, it is possible that caffeine's link to ischemic events could be due to the physiological response it engenders by its ergogenic effects in lieu of its role in clotting potential.

Although the CYP1A2 polymorphism did not influence the Factor VIII response to caffeine, $\mathrm{C}$ allele carriers exhibited a greater plasma fibrinogen response after ingesting caffeine compared to AA homozygotes. Carriers of the $\mathrm{C}$ allele exhibited a $23 \%$ increase in post-exercise fibrinogen values after ingesting caffeine, whereas the AA homozygotes showed an increase of less than $0.1 \%$. This difference was apparent even after co-varying for delta TTF, suggesting that the increased time to fatigue engendered by the caffeine did not influence this response. Prior studies on the fibrinogen response to exercise have been equivocal as increases [3], decreases [5,51] and static responses [31,52,56] have been observed [8,52]. Furthermore, prior studies have observed an association between coffee consumption and myocardial infarction in $\mathrm{C}$ allele carriers, but not AA homozygotes [16,17]. It is possible that this observation is at least partially linked to an increase in fibrinogen in response to physical stress in the $\mathrm{C}$ allele carriers. While the present study does not definitively support this contention, it is compelling that this was the only hemostatic marker investigated that demonstrated a significant treatment $\mathrm{x}$ time $\mathrm{x}$ genotype interaction.

During exercise, the increased coagulation potential is accompanied by a corresponding increase in fibrinolytic potential $[1,11,14,26,28,38,39]$. Plasma tPA activity has been implicated as a strong predictor for acute myocardial infarction in many studies [46,47]. tPA is a precursor for the catalyzing process of plasminogen to plasmin, the active enzyme directly responsible for the lysis of fibrin clot. However, after periods of strenuous exercise, markers of both coagulation and fibrinolysis do not return to resting levels at similar rates [50]. In fact, markers of 
coagulation have been reported to stay elevated anywhere from 6-10 hours after the cessation of exercise while markers of fibrinolytic potential have been reported to return to resting levels in as few as 10 minutes [28,39,50]. Regardless of whether the increase in post-exercise Factor VIII was influenced by test duration, it was counterbalanced by a greater increase in tPA during the caffeinated condition. tPA increased 20-fold after exercise for both caffeine and placebo trials, supporting the idea of a counterbalanced activity of coagulation immediately after periods of maximal exertion. However, a significantly greater rise in post-exercise tPA levels was observed with the caffeine treatment $($ Caffeine $=9.59 \mathrm{IU} / \mu 1$; Placebo $=6.89 \mathrm{IU} / \mu 1, \mathrm{p}<0.05)$, suggesting that the increased coagulation potential after exercise in the caffeinated condition was counter-balanced by increases in fibrinolytic potential. In contrast to Factor VIII, however, the treatment and treatment $\mathrm{x}$ time effect for caffeine remained significant when covarying for the difference in time to fatigue. Some studies have suggested a potential protective effect for CVD with caffeine consumption $[16,29,45,48,53]$. As the present study showed an increase in tPA with caffeine consumption, both at rest and in response to stress, it is possible that these aforementioned effects are at least partially due to improvements in fibrinolytic potential. The main circulating inhibitor of tPA, PAI-1 was not significantly affected by either caffeine or exercise. While PAI-1 is known to decrease as a response to exercise [15], an absence of any response has also been observed [9]. Thus, in the present study, the impact of caffeine on fibrinolysis appeared to be confined to increases in tPA activity.

The CYP1A2 polymorphism did not affect $\mathrm{VO}_{2 \text { peak }}$ or TTF in the current study. In contrast, a study from our lab showed that caffeine supplementation had a more pronounced ergogenic effect on $40 \mathrm{~km}$ time trial performance in trained cyclists homozygous for the A allele [60]. However, it should be realized that the current study utilized a graded exercise test and the 
participants were not trained cyclists. It could be that the lack of genetic effect is specific to the protocol and/or sample group used in the present study.

While the findings from the present study suggest a hemostatic response from caffeine and a genetic influence on this hemostatic response, there are some caveats that should be noted. Our study of healthy, college-aged males did not include analysis of any clinical population and so our results cannot be extended to populations that may have increased coagulation potential and/or decreased fibrinolysis. In addition, it has been proposed that hormonal fluctuations during the menstrual cycle may affect coagulation and fibrinolysis, at least acutely, and so our results may not be accurately translated to females. Also, there was no post-exercise period of measurements taken from subjects other than those upon immediate cessation of the maximal exercise test. Thus, it is not known the degree to which caffeine affected these markers in the post-exercise period and to what extent the studied polymorphism influenced any effect. The area of research regarding caffeine's relationship to ischemic events is extremely limited as the majority of literature is in regards to coffee and cardiovascular disease risk. It is because of this that our results may not be indicative of the effect that coffee has on coagulation potential. Lastly, the subject number used in this study is substantially less than that of what is typically used in studies regarding polymorphisms and genetic differences. Thus it is possible that not enough participants were observed to detect some of the potential genetic differences. However, no trends were evident for either genotype $\mathrm{x}$ treatment or genotype $\mathrm{x}$ treatment $\mathrm{x}$ time interactions for any of the variables examined in the present study. In summary, the present study suggests that caffeine increases resting and post-exercise tPA activity levels. Furthermore, caffeine may increase Factor VIII response to graded exercise secondary to an effect on time to fatigue. The CYP1A2 A/C polymorphism appears to impact how caffeine influences hemostatic 
response to exercise; as only $\mathrm{C}$ allele carriers exhibited a significant increase in fibrinogen during exercise in the caffeinated condition. Future research should evaluate the link between this genetic effect and if it influences risk for ischemic events. 


\section{References}

1. Andrew, M., Carter, C., O’Brodovich, H., \& Heigenhauser, G. Increases in factor VIII complex and fibrinolytic activity are dependent on exercise intensity. Journal of Applied Physiology. 1986; 60(6):1917-22.

2. Angleton, P., Chandler, W. L., \& Schmer, G. Diurnal variation of tissue-type plasminogen activator and its rapid inhibitor (PAI-1). Circulation. 1989;79(1): 101-106.

3. Arai M, Yorifuji H, Ikematsu S, Nagasawa H, Fujimaki M, Fukutake K, Katsumura T, Ishii T, Iwane H. Influences of strenuous exercise (triathlon) on blood coagulation and fibrinolytic system. Thromb Res. 1990;57(3):465-71.56.

4. Bak, A. A. A., Van Vliet, H. H. D. M., \& Grobbee, D. E.. Coffee, caffeine and hemostasis: Results from two randomized studies. Atherosclerosis. 1990; 83(2-3): 249255.

5. Bärtsch P, Haeberli A, Straub PW. Blood coagulation after long distance running: antithrombin III prevents fibrin formation. Thromb Haemost. 1990;63(3):430-4.

6. Baylin A, Hernandez-Diaz S, Kabagambe EK, Siles X, Campos H. Transient exposure to coffee as a trigger of a first nonfatal myocardial infarction. Epidemiology. 2006;17(5):506-511.

7. Bender, a M., Donnerstein, R. L., Samson, R. a, Zhu, D., \& Goldberg, S. J. Hemodynamic effects of acute caffeine ingestion in young adults. The American Journal of Cardiology. 1997; 79(5):696-9. 
8. Bizheh, N., \& Jaafari, M. The effect of a single bout circuit resistance exercise on homocysteine, hs-CRP and fibrinogen in sedentary middle aged men. Iranian Journal of Basic Medical Sciences. 2011;14(6):568-573.

9. Bodary, P. F., Yasuda, N., Watson, D. D., Brown, A. S., Davis, J. M., \& Pate, R. R.. Effects of short-term exercise training on plasminogen activator inhibitor (PAI-1). Medicine and Science in Sports and Exercise. 2003;35(11): 1853-8.

10. Bøhn, S. K., Ward, N. C., Hodgson, J. M., \& Croft, K. D. Effects of tea and coffee on cardiovascular disease risk. Food \&Function. 2012; 3(6): 575-91.

11. Burg PJ van den, Hospers JE, van Vliet M, Mosterd WL, Bouma BN, Huisveld IA. Effect of endurance training and sea- sonal fluctuation on coagulation and fibrinolysis in young sedentary men. J Appl Physiol. 1997; 82: 613-620.

12. Cai L, Ma D, Zhang Y, Liu Z, Wang P. The effect of coffee consumption on serum lipids: A meta-analysis of randomized controlled trials. Eur J Clin Nutr. 2012;66(8):872877.

13. Casiglia, E., Paleari, C. D., Petucco, S., Bongiovi, S., Colangeli, G., Baccilieri, M. S., Pessina, A. C. Haemodynamic effects of coffee and purified caffeine in normal volunteers: A placebo-controlled clinical study. Journal of Human Hypertension. 1992;6(2): 95-99.

14. Chandler WL, Veith RC, Fellingham GW, Levy WC, Schwartz RS, Cerqueira MD, Kahn SE, Larson VG, Cain KC, Beard JC, et al. Fibrinolytic response during exercise and epinephrine infusion in the same subjects. J Am Coll Cardiol. 1992;19:1412-1420. 
15. Cooper, J. a., Nagelkirk, P. R., Coughlin, A. M., Pivarnik, J. M., \& Womack, C. J. Temporal Changes in tPA and PAI-1 after Maximal Exercise. Medicine \& Science in Sports \& Exercise. 2004;36(11), 1884-1887.

16. Cornelis, M. C., A. El-Sohemy, and H. Campos. Genetic Polymorphism of CYP1A2 Increases the Risk of Myocardial Infarction. Journal of Medical Genetics. 2004;41(10): 758-62.

17. Cornelis, M. C., et al. Coffee, CYP1A2 Genotype, and Risk of Myocardial Infarction. Journal of the American Medical Association. 2006;295(10): 1135-41.

18. Corrêa, T. a F., Rogero, M. M., Mioto, B. M., Tarasoutchi, D., Tuda, V. L., César, L. a M., \& Torres, E. a F. S. Paper-filtered coffee increases cholesterol and inflammation biomarkers independent of roasting degree: a clinical trial. Nutrition. 2013;29(7-8):97781.

19. Curatolo, P. W., \& Robertson, D.. The health consequences of caffeine. Annals of Internal Medicine. 1983;98(5 Pt 1):641-53.

20. Davis GL, Abildgaard CF, Bernauer EM, Britton M. Fibrinolytic and hemostatic changes during and after maximal exercise in males. J Appl Physiol. 1976;40(3):287-92.

21. El-Sayed MS, Sale C, Jones PG, Chester M. Blood hemostasis in exercise and training. Med Sci Sports Exerc. 2000;32(5):918-25.

22. Farag, N. H., Vincent, A. S., Mckey, B. S., Whitsett, T. L., \& Lovallo, W. R. Hemodynamic Mechanisms Underlying the Incomplete Tolerance to Caffeine's Pressor Effects. American Journal of Cardiology. 2005;1389-1392. 
23. Freedman, N. D., Park, Y., Abnet, C. C., Hollenbeck, A. R., \& Sinha, R. Association of coffee drinking with total and cause-specific mortality. New England Journal of Medicine. 2012;366(20):1891-1904.

24. Giri S, Thompson PD, Kiernan FJ, et al. Clinical and Angiographic Characteristics of Exertion-Related Acute Myocardial Infarction. JAMA. 1999;282(18):1731-1736.

25. Greenland S. A meta-analysis of coffee, myocardial infarction, and coronary death. Epidemiology. 1993;4(4):366-74

26. Groen WG, den Uijl IE, van der Net J, Grobbee DE, de Groot PG, Fischer K. Protected by nature? Effects of strenuous physical exercise on FVIII activity in moderate and mild haemophilia A patients: a pilot study. Haemophilia. 2013;19(4):519-23.

27. Hallström, H., Melhus, H., Glynn, A., Lind, L., Syvänen, A.-C., \& Michaëlsson, K. Coffee consumption and CYP1A2 genotype in relation to bone mineral density of the proximal femur in elderly men and women: a cohort study. Nutrition \& Metabolism. $2010 ; 7(12)$

28. Hansen JB, Wilsgard L, Olsen JO, Osterud B. Formation and persistence of procoagulant and fibrinolytic activities in circulation after strenuous physical exercise. Thromb Haemost, 1990;64:385-389

29. Happonen P, Voutilainen S, Salonen JT. Coffee drinking is dose-dependently related to the risk of acute coronary events in middle-aged men. J Nutr. 2004;134(9):2381-2386.

30. Heinrich, J., Balleisen, L., Schulte, H., Assmann, G., \& van de Loo, J. Fibrinogen and factor VII in the prediction of coronary risk. Results from the PROCAM study in healthy men. Arteriosclerosis, Thrombosis, and Vascular Biology. 1994;14(1):54-59. 
31. Herren T, Bärtsch P, Haeberli A, Straub PW. Increased thrombin-antithrombin III complexes after 1 h of physical exercise. J Appl Physiol. 1985;73(6):2499-504.

32. Jee, S. H., He, J., Appel, L. J., Whelton, P. K., Suh, I., \& Klag, M. J. Coffee Consumption and Serum Lipids : A Meta-Analysis of Randomized Controlled Clinical Trials. American Journal of Epidemiology. 2001;153(4):353-362.

33. Jee SH, He J, Whelton PK, Suh I, Klag MJ. The effect of chronic coffee drinking on blood pressure: A meta-analysis of controlled clinical trials. Hypertension. 1999;33(2):647-652.

34. Kawachi I, Colditz GA, Stone CB. Does coffee drinking increase the risk of coronary heart disease? results from a meta-analysis. Br Heart J. 1994;72(3):269-275.

35. Kim B, Nam Y, Kim J, Choi H, Won C. Coffee consumption and stroke risk: A metaanalysis of epidemiologic studies. Korean Journal of Family Medicine. 2012;33(6):356365.

36. Kleemola, P., Jousilahti, P., Pietinen, P., Vartiainen, E., \& Tuomilehto, J. Coffee consumption and the risk of coronary heart disease and death. Archives of Internal Medicine. 2014;160(22): 3393-400.

37. Kovanen, P. T., \& Pentika, M. O. Pharmacologic prevention of coronary plaque rupture the major cause of acute coronary syndromes. Heart Metabolism. 2007;36:9-14.

38. Lee CW, Ahn JM, Park DW, Kim YH, Hong MK, Song JK, Kim JJ, Park SW, Chi HS, Park SJ. Tissue plasminogen activator on admission is an important predictor of 30-day mortality in patients with acute myocardial infarction undergoing primary angioplasty. Atherosclerosis. 2008;196(1):327-32. 
39. Lin X, El-Sayed MS, Waterhouse J, Reilly T. Activation and disturbance of blood haemostasis following strenuous physical exercise. Int J Sports Med. 1999;20:149-153.

40. Liu J, Sui X, Lavie CJ, Hebert JR, Earnest CP, Zhang J, \& Blair SN. Association of coffee consumption with all-cause and cardiovascular disease mortality. Mayo Clinic Proceedings. 2013;88(10): 1066-74.

41. Lopez-Garcia, E., Dam, R. M. Van, Li, T. Y., Rodriguez-Artalejo, F., \& Hu, F. B. The Relationship of Coffee Consumption with Mortality. Annals of Internal Medicine. 2013;147(12):904-14.

42. Lovallo W, Wilson M, Vincent A. Blood pressure response to caffeine shows incomplete tolerance after short-term regular consumption. Hypertension. 2004;43(4):760-765.

43. Mort JR, Kruse HR. Timing of blood pressure measurement related to caffeine consumption. Ann Pharmacother. 2008;42(1):105-10.

44. Mostofsky E, Schlaug G, Mukamal KJ, Rosamond WD, Mittleman MA. Coffee and acute ischemic stroke onset: The stroke onset study. Neurology. 2010;75(18):1583-1588.

45. Natella F, Nardini M, Belelli F, et al. Effect of coffee drinking on platelets: Inhibition of aggregation and phenols incorporation. Br J Nutr. 2008;100(6):1276-1282.

46. Niessner A, Graf S, Nikfardjam M, Speidl WS, Huber-Beckmann R, Zorn G, Wojta J, Huber K. Circulating t-PA antigen predicts major adverse coronary events in patients with stable coronary artery disease--a 13-year follow-up. Thromb Haemost. 2003;90(2):344-50.

47. Nordenhem A, Wiman B. Tissue plasminogen activator (tPA) antigen in plasma: correlation with different tPA/inhibitor complexes. Scand J Clin Lab Invest. 1998;58(6):475-83. 
48. Panagiotakos DB, Pitsavos C, Chrysohoou C, Kokkinos P, Toutouzas P, Stefanadis C. The J-shaped effect of coffee consumption on the risk of developing acute coronary syndromes: The CARDIO2000 case-control study. J Nutr. 2003;133(10):3228-3232.

49. Papaioannou, T. G., Karatzi, K., Karatzis, E., Papamichael, C., \& Lekakis, J. P. Acute effects of caffeine on arterial stiffness, wave reflections, and central aortic pressures. American journal of hypertension. 2005:18(1):129-36.

50. Paton, C. M., Nagelkirk, P. R., Coughlin, a M., Cooper, J. a, Davis, G. a, Hassouna, H., Womack, C. J. Changes in von Willebrand factor and fibrinolysis following a postexercise cool-down. European journal of applied physiology. 2004;92(3):328-33.

51. Prisco D, Paniccia R, Bandinelli B, Fedi S, Cellai AP, Liotta AA, Gatteschi L, Giusti B, Colella A, Abbate R, Gensini GF. Evaluation of clotting and fibrinolytic activation after protracted physical exercise. Thromb Res. 1998;89(2):73-8.

52. Rankinen, T., Valsanen, S., Penttila, I., \& Rauramaa, R. Acute dynamic exercise increases fibrinolytic activity. Thrombosis and Haemostasis. 1995;73(2): 281-286.

53. Shah, P. K. Mechanisms of plaque vulnerability and rupture. Journal of the American College of Cardiology. 2003;41(4): S15-S22.

54. Sofi, F., Conti, A. a, Gori, A. M., Eliana Luisi, M. L., Casini, A., Abbate, R., \& Gensini, G. F. Coffee consumption and risk of coronary heart disease: a meta-analysis. Nutrition, metabolism, and cardiovascular diseases. 2007;17(3):209-23.

55. Strandhagen, E., \& Thelle, D. S. Filtered coffee raises serum cholesterol: results from a controlled study. European journal of clinical nutrition. 2003;57(9):1164-8.

56. Watts EJ. Haemostatic changes in long-distance runners and their relevance to the prevention of ischaemic heart disease. Blood Coagul Fibrinolysis. 1991;2(2):221-5. 
57. Williams, R. B., \& Kuhn, C. M. Caffeine Effects on Cardiovascular and Neuroendocrine Responses to Acute Psychosocial Stress and Their Relationship to Level of Habitual Caffeine Consumption. Psychosomatic Medicine. 1990;336:320-336.

58. Winther K, Hillegass W, Tofler GH, Jimenez A, Brezinski DA, Schafer AI, Loscalzo J, Williams GH, Muller JE. Effects on platelet aggregation and fibrinolytic activity during upright posture and exercise in healthy men. American Journal of Cardiology. 1992;70:1051-1055

59. Womack, C. J., Rasmussen, J. M., Vickers, D. G., Paton, C. M., Osmond, P. J., \& Davis, G. L. Changes in fibrinolysis following exercise above and below lactate threshold. Thrombosis research. 2006;118(2):263-8.

60. Womack, C. J., Saunders, M. J., Bechtel, M. K., Bolton, D. J., Martin, M., Luden, N. D., Dunham, W., et al. The influence of a CYP1A2 polymorphism on the ergogenic effects of caffeine. Journal of the International Society of Sports Nutrition, 2012;9(1):7. 